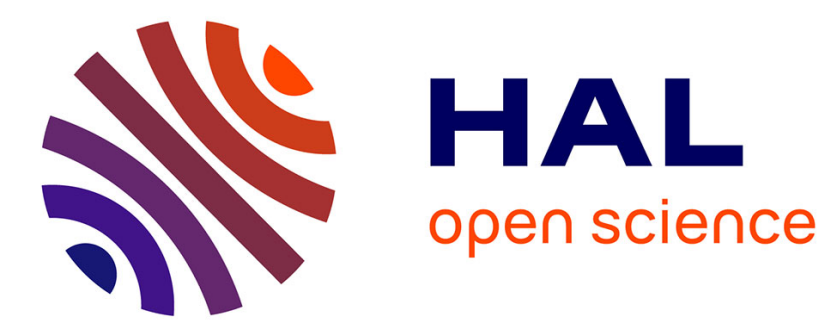

\title{
La commercialisation du service d'eau potable à Windhoek (Namibie) : inégalités urbaines et logiques marchandes
}

Sylvy Jaglin

\section{- To cite this version:}

Sylvy Jaglin. La commercialisation du service d'eau potable à Windhoek (Namibie) : inégalités urbaines et logiques marchandes. Flux - Cahiers scientifiques internationaux Réseaux et territoires, 1997, 13 (30), pp.16 - 29. 10.3406/flux.1997.1212 . halshs-03253644

\section{HAL Id: halshs-03253644 \\ https://shs.hal.science/halshs-03253644}

Submitted on 8 Jun 2021

HAL is a multi-disciplinary open access archive for the deposit and dissemination of scientific research documents, whether they are published or not. The documents may come from teaching and research institutions in France or abroad, or from public or private research centers.
L'archive ouverte pluridisciplinaire HAL, est destinée au dépôt et à la diffusion de documents scientifiques de niveau recherche, publiés ou non, émanant des établissements d'enseignement et de recherche français ou étrangers, des laboratoires publics ou privés. 


\section{La commercialisation du service d'eau potable à Windhoek} (Namibie) : inégalités urbaines et logiques marchandes Sylvy Jaglin

\section{Citer ce document / Cite this document :}

Jaglin Sylvy. La commercialisation du service d'eau potable à Windhoek (Namibie) : inégalités urbaines et logiques marchandes. In: Flux, n³0, 1997. pp. 16-29;

doi : https://doi.org/10.3406/flux.1997.1212

https://www.persee.fr/doc/flux_1154-2721_1997_num_13_30_1212

Fichier pdf généré le 12/05/2018 


\begin{abstract}
The commercialization of the Namibian Department of Water Affairs is an institutional answer to a double problem: the shortage of both water and public funds in the face of increasing urban demand. This practice aims at bringing the legal statutes and the practices of bulk water supply management into compliance with the commercial principles of this sector of activity. However, in a context characterized by increasing and evolving post-apartheid intra-urban inequalities, in which a small Black elite does not yet possess the technical control of the major urban services, combined with disparities in the implementation process of recent decentralization, this institutional reform seems inadequate to contend with two sizeable challenges: managing water shortage as well as sharing the resource among users in a sustainable and thrifty way; providing the urban poor with an adequate supply for which the technical and financial arrangements remain to be invented.
\end{abstract}

\title{
Résumé
}

La commercialisation du Department of Water Affairs namibien est une réponse institutionnelle à une double difficulté : la raréfaction de la ressource naturelle et celle des financements publics face à une demande urbaine croissante. Elle vise à conformer le statut juridique et les pratiques de l'organisme gestionnaire de l'eau en gros aux logiques commerciales de ce secteur d'activité. Dans un contexte marqué par l'accroissement et la transformation des inégalités intra-urbaines post-apartheid, l'étroitesse d'une élite politique noire encore très largement privée du commandement technique des grands services urbains et les disparités de mise en œuvre d'une décentralisation encore fragile, cette réforme institutionnelle semble toutefois insuffisante pour relever les deux grands défis auxquels est confronté le service d'eau urbain : gérer la pénurie et, partant, le partage de la ressource d'une façon plus durable et économe ; assurer la desserte de clientèles urbaines très pauvres pour lesquelles des modes d'approvisionnement et de financement spécifiques restent à inventer. 
FLUX $n^{\circ} 30$ Octobre-Décembre 1997

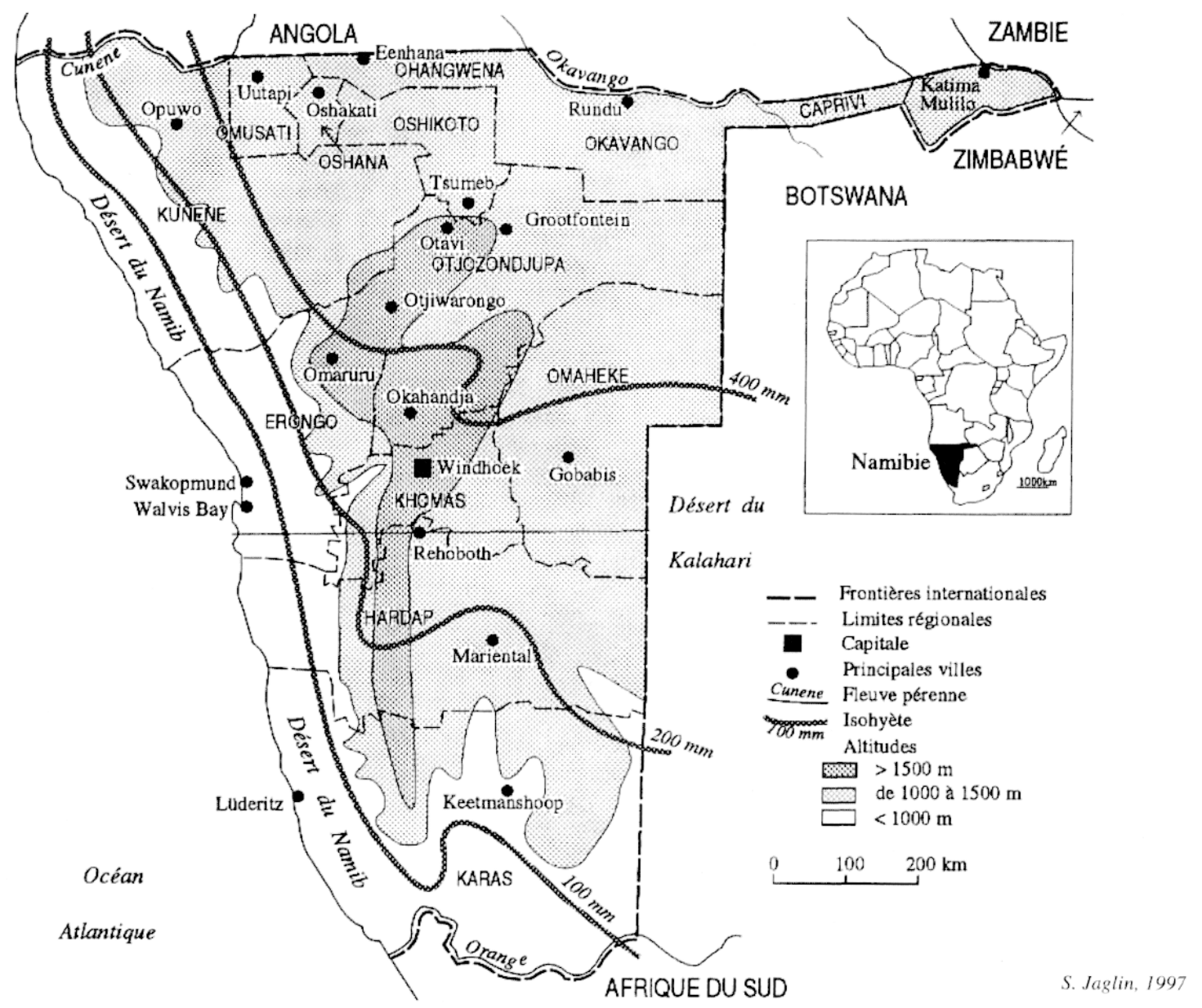

LA NAMIBIE 


\section{FLUX}

$n^{\circ} 30$

Octobre - Décembre

1997

pp. $16-29$
La commercialisation du service d'eau potable à Windhoek
(Namibie) : inégalités urbaines et
logiques marchandes

SYLVY JAGLIN
Sylvy JAGLIN, géographe-urbaniste. est maître de conférences à l'Institut français d'urbanisme (Université Paris VIII) et membre de l'UMR 7543 Département Théorie des mutations urbaines. Ses recherches actuelles portent sur l'évolution de la gestion urbaine dans les villes ségréguées d'Afrique australe. Il s'agit en particulier d'analyser, dans un contexte de décentralisation et de privatisation, les conséquences des mutations des services publics sur des sociétés citadines qu'affecte une polarisation croissante.
C APITALE D'UN PAYS SEMI-ARIDE, Windhoek doit son site, à $1700 \mathrm{~m}$ d'altitude, à la présence de sources chaudes pérennes. Principalement assuré, de 1912 à 1959, par des forages municipaux, son approvisionnement en eau l'est, depuis, par des barrages étatiques et une station de retraitement ${ }^{1}$. La nécessité stratégique de maitriser la ressource en eau est ancienne et les dispositifs inventés pour y répondre sont, à ce titre, révélateurs des grands équilibres sociétaux qui ont prévalu en Namibie depuis le début du $\mathrm{xx}^{\mathrm{e}}$ siècle.

Tant que l'usage de l'eau, d'abord destinée aux grandes fermes d'élevage puis à une clientèle urbaine blanche privilégiée par le régime d'apartheid, ne fut guère contesté, la quête de cette ressource a mobilisé la mise en œuvre de moyens techniques sophistiqués sans susciter ni problèmes financiers ni graves conflits d'arbitrage. Il en va aujourd'hui tout autrement : non que la tension actuelle entre offre et demande soit nouvelle ou surprenante, mais elle survient alors que l'indépendance politique de 1990 accélère le démantèlement d'un apartheid officiellement abandonné en 1977 et que les réponses institutionnelles à la rareté en eau sont profondément modifiées par les contraintes financières du nouvel État namibien comme par l'évolution des doctrines économiques en matière de services marchands.

Ainsi, l'impérieuse nécessité d'une réforme résulte moins d'une crise du système antérieur de gestion que d'une refonte, symboliquement importante, d'un grand service public lié, dans son organisation comme dans son fonctionnement, à l'ancien régime d'apartheid : l'obsolescence du dispositif antérieur de régulation (au sens d'organisation d'ensemble du cadre d'exploitation et d'usage de l'eau) impose de reconcevoir la place de ce service dans les villes. Ce faisant, le processus de mutation n'échappe ni à l'influence de l'idéologie démocratico-libérale prégnante dans toutes les restructurations en cours dans les pays en développement, ni au savoir-faire stéréotypé des experts internationaux. Sera-t-il néanmoins attentif aux deux grands défis du service urbain de l'eau à Windhoek : sa modernisation gestionnaire, dans un contexte de raréfaction de l'eau et des capitaux publics, et sa généralisation, politiquement cruciale, à l'ensemble des citadins alors que s'enracinent en ville des contingents croissants de populations non solvables? 


\section{DE L'EAU CONFISQUÉE À L'EAU PARTAGÉE : UNE TRANSITION DIFFICILE}

\section{"L'APARTHEID HYDRIQUE » : UN SYSTĖME DE RÉGULATION ANACHRONIQUE}

Pour satisfaire la demande des citadins blancs, l'eau est fournie en abondance dans les quartiers résidentiels à faible densité où piscines et jardins luxuriants témoignent des réussites individuelles avec des excès de démonstration à la mesure des inégalités sociales : les mécanismes de l'apartheid ont en effet concentré la richesse et les services de grande qualité au profit d'une minorité et conduit à une distribution des revenus qui figure parmi les plus inégales du monde, $5 \%$ de la population contrôlant $71 \%$ du PNB à la fin des années 80 (World Bank, 1992).

Il n'est donc guère surprenant que les consommations dans les beaux quartiers, principalement dues au jardinage, soient exceptionnellement élevées : $615 \mathrm{l} / \mathrm{h} / \mathrm{j}$ en moyenne pour les 32720 habitants de la City. Les autres groupes de résidants, qui représentent presque $80 \%$ de la population, ont des pratiques plus parcimonieuses, responsables d'à peine $41 \%$ de la consommation d'eau domestique. Soulignons encore que les plus pauvres $(50 \%$ de la population) consomment à peine $15 \%$ de l'eau distribuée aux ménages à Windhoek.
Comme l'ensemble des ressources disponibles, l'eau a été gérée par la société d'apartheid en vertu du principe d'exclusion, l'abondance étant préservée pour la minorité blanche grâce à l'exclusion partielle de la majorité noire. Cet « apartheid hydrique » s'est traduit par des disparités très importantes dans les consommations spécifiques, dont les plafonds sont étroitement corrélés aux niveaux de vie. De tels déséquilibres sont aujourd'hui remis en cause, l'accès à l'eau étant un enjeu majeur de l'intégration urbaine des Noirs et les ressources disponibles ne permettant pas de satisfaire toutes les nouvelles demandes en maintenant les comportements antérieurs.

\section{LA FIN D'UN SYSTÈME OU LE TERRITOIRE ÉCARTELÉ}

Les estimations les plus optimistes font état d'une demande en augmentation de $5 \%$ par an à Windhoek jusqu'en 2020 , ce qui suppose d'infléchir notablement une croissance établie à $6,5 \%$ par an entre 1985 et 1990 , mais souvent voisine de $8 \%$ par an dans le passé. Toutes les ressources en eau disponibles à proximité de Windhoek sont exploitées et aucune augmentation substantielle de la production n'est envisageable sans d'importants investissements pour assurer des transferts longue distance. La ville est donc dans une période de raréfaction de la ressource ce qui, historiquement, n'est pas inédit. L'installation durable dans une situation de précaire équilibre est en

Table 1. Répartition des consommations d'eau à Windhoek en 1991 


\section{Jaglin - Le service d'eau potable à Windhoek}

revanche nouvelle : trois catégories de facteurs se conjuguent pour expliquer cette aggravation.

\section{Des transferts inter-régionaux toujours plus coûteux}

La dilatation des bassins d'exploitation implique de coûteux transferts inter-régionaux : les travaux nécessaires à l'approvisionnement de la capitale et de la région centrale jusqu'en 2020 ont ainsi été évalués à N\$ 2,4 milliards ${ }^{2}$ (coûts 1993). Ils comprennent notamment l'exploitation des nappes phréatiques du TGO Triangle (TsumebGrootfontein-Otavi) et une liaison avec l'Okavango, à 750 $\mathrm{km}$ au nord. Au-delà des dépenses d'investissement et de l'endettement de l'État namibien, de tels projets engendreront d'importants coûts de gestion et un surenchérissement du prix de l'eau : la municipalité estimait ainsi en 1995 que si les seuls investissements nécessaires à l'établissement de l'adduction entre l'Okavango et Windhoek étaient répercutés sur le prix d'achat de l'eau en gros, celui-ci augmenterait de $\mathrm{N} \$ 1,24 / \mathrm{m}^{3}$ à $\mathrm{N} \$ 9,43 / \mathrm{m}^{3}$ (Windhoek Aloe, $\mathrm{n}^{\circ} 5.3,1995$ ).

Ces incidences économiques ne sont pas seules en cause dans l'émergence d'une nouvelle gestion de la ressource : la sensibilité accrue des milieux politiques aux questions environnementales et au développement durable les conduit à dénoncer, au moins dans les discours, les consommations somptuaires de certaines catégories de citadins. En outre, le pouvoir namibien ne peut rester insensible aux inquiétudes des pays voisins, qui dépendent en partie des mêmes ressources en eau (l'Okavango dessert le Botswana et l'Angola et, pour l'heure, aucun accord régional n'a été établi quant à l'utilisation du fleuve par les États bordiers), comme à celles des écologistes qui défendent les écosystèmes fragiles des rives de l'Okavango et du Cunene. Il faut donc adapter une administration de prospection et de croissance à de nouvelles tâches de conservation et de répartition.

\section{Forte croissance urbaine et polarisation accrue du territoire national}

Windhoek concentre à la fois richesse et opportunités d'emplois (40\% de l'emploi formel et $39 \%$ des établissements productifs y sont localisés) : elle est un pôle majeur d'attraction, notamment pour les régions septentrionales densément peuplées et écologiquement dévastées par la guerre et les sécheresses successives. Avec une population estimée à 200000 habitants, dont $50 \%$ de migrants, au milieu des années 90 et des taux de croissance démographique de $6 \%$ à $8 \% / \mathrm{an}^{3}$, elle rassemble plus du tiers de la population urbaine namibienne. Il est peu vraisemblable que cette concentration économique et démographique au profit de la capitale s'inverse à moyen terme. L'État semble en effet dépourvu d'un projet cohérent de redistribution de la croissance économique sur le territoire et d'encadrement de l'urbanisation à l'échelle nationale (Melber 1996 ; NEPRU 1997), tandis que la municipalité multiplie les initiatives pour soutenir la croissance locale, démontrant que la crainte d'une pénurie d'eau ne bride nullement ses ambitions dans une compétition économique accrue entre les villes moyennes de la SADC.

Cette évolution a deux conséquences pour la gestion de l'eau : elle radicalise la distorsion géographique entre les lieux de l'expansion économique nationale, générateurs d'une forte demande en eau, et les sites pourvoyeurs de ressources en eau ; elle aggrave la compétition entre les usagers urbains mais aussi, au sein de la région centra$l^{4}$, entre le principal consommateur - la municipalité de Windhoek - et les autres utilisateurs (mines, agriculteurs, game farms).

\section{Des partenariats à recomposer}

L'approvisionnement en eau potable des villes namibiennes relève, historiquement, d'un service public qu'animent deux principaux acteurs : une direction ministérielle détentrice du monopole public de la production/distribution de l'eau en gros et des pouvoirs locaux responsables de la desserte de proximité. À Windhoek, le dispositif acquis à l'indépendance est le suivant :

- le Department of Water Affairs produit en moyenne $75 \%$ à $80 \%$ de l'eau distribuée dans la capitale, facturés à la municipalité en fonction d'un tarif révisé et approuvé chaque année qui doit assurer, au minimum, le recouvrement des coûts de fonctionnement. Jusqu'à présent, la modernisation et l'extension des réseaux urbains d'infrastructures ont été financièrement assumées par l'État.

- le City Engineer's Department gère le système réticulaire de distribution sur le territoire communal et produit environ $25 \%$ de l'eau distribuée. Pour ses clients, la municipalité établit des tarifs qui comprennent l'achat de 
l'eau en gros, les coûts de production à partir des installations municipales et ceux de la distribution locale : ils permettent un recouvrement total de l'ensemble des charges induites (fonctionnement et investissements).

Ce dispositif, repris avec quelques variantes dans les autres municipalités, a permis d'assurer le contrôle de l'État sur une ressource rare et coûteuse tout en laissant aux citadins blancs, représentés par le conseil municipal, les moyens de peser sur l'organisation du service de proximité. Sa disqualification résulte d'une fragilisation et d'une contestation des partenariats sur lesquels était fondée jusqu'alors la gestion.

En effet, le passage d'une politique d'expansion à une gestion de pénurie ébranle la coopération entre la municipalité de Windhoek et le Department of Water Affairs. La première dispose de ressources qui dépendent notamment de la vente des services locaux (eau et électricité) dont elle soutient la croissance. Le second assume les principales dépenses d'infrastructure dans un contexte de concurrence accrue entre ses différents clients, d'étiage des subventions étatiques et de rationalisation gestionnaire. Les arbitrages entre le producteur d'eau en gros et ses clients sont ainsi à redéfinir.

De façon plus générale, la modernisation de l'administration publique enclenchée par l'État namibien modifie les relations entre ces acteurs. D'une part la décentralisation, avec le Local Authorities Act de 1992, impose une clarification des responsabilités entre échelons territoriaux, de sorte que la puissante forteresse du Department of Water Affairs est aujourd'hui perçue comme une entrave au processus de consolidation des pouvoirs locaux. D'autre part l'introduction des ferments du marché dans la sphère publique remet en cause la culture bureaucratique antérieure (notamment fondée sur une confusion entre le caractère d'intérêt général du service urbain de l'eau et le statut public de l'organisme gestionnaire, ainsi que sur une pratique étendue de péréquation et de subvention à la production), considérée comme un obstacle au développement d'une économie des services « efficace ».

Enfin, le binôme Department of Water Affairs /municipalités a fonctionné, jusqu'en 1992, dans le cadre de villes blanches (flanquées de leur township métisse), dotées de compétences techniques et financières qui leur permettaient d'assurer les services locaux sans s'encombrer des contraintes sociales et économiques de la desserte des pauvres. L'intégration des townships noires dans les territoires d'administration des collectivités locales reporte sur ces dernières la totalité de la charge financière et gestionnaire des services aux populations déshéritées, alors que tarissent les subventions étatiques.

Pour rompre avec l'organisation antérieure, il s'agit de transformer des systèmes gestionnaires producteurs d'inégalités en systèmes capables de les atténuer. Est-ce aux organismes gestionnaires de l'eau d'endosser cette responsabilité ? Est-ce aux pouvoirs publics?

\section{EN QUÊTE DE NOUVEAUX COMPROMIS ENTRE EFFICACITÉ ÉCONOMIQUE ET UTILITÉ SOCIALE}

En partie sous l'impulsion d'un cabinet de consultance international, la réforme institutionnelle s'inspire de principes bien connus : injection de règles du marché dans le monopole public, décentralisation des fonctions de pilotage dans les systèmes gestionnaires au plus près des lieux de desserte, mobilisation des usagers/consommateurs du service. Prompt à déceler les défaillances du système public, son argumentaire l'est autant dans sa litanie des avantages supposés de la commercialisation, évoqués au travers de comparaisons internationales (Grande Bretagne, Nouvelle Zélande...) dont la pertinence est douteuse, mais il évite soigneusement des questions cruciales.

Quelles sont les ressources, notamment politiques, dont dispose la société namibienne pour imposer de nouveaux critères d'efficacité productive, à l'amont de changements durables dans les services publics ? On se fait volontiers à l'idée du conservatisme de l'establishment blanc, mais apprécie-t-on avec justesse les capacités d'innovation du nouveau leadership noir ? Ne sous-estime-t-on pas des formes de résistance issues, par exemple, de sa méconnaissance des mécanismes d'un pouvoir dont il a été évincé si longtemps ou du souci politique de privilégier le rattrapage et la compensation au profit des plus défavorisés, ce qui conduit à reproduire les savoirs existants plutôt qu'à promouvoir des solutions inventives ?

Dans quelle mesure la pauvreté urbaine, celle qui 
frappe notamment les citadins noirs des périphéries, ne peut-elle faire obstacle à la réforme ? Comment surmonter le dualisme antérieur de la société d'apartheid et maximiser l'utilité sociale du service sans tomber dans les errances d'une gestion dispendieuse et insupportable sur le long terme par les budgets publics?

\section{LA NOUVELLE POLITIQUE DE L'EAU}

L'eau et l'assainissement sont régis par une nouvelle politique sectorielle, la WASP (Water and Sanitation Policy), adoptée le 21 septembre 1993 (MAWRD/DWA, 1993-b), qui définit l'eau comme une marchandise : son exploitation doit revêtir un caractère durable dans un cadre institutionnel qui, sans exclure l'État, l'incite progressivement à se départir de son rôle de producteur direct (provider) au profit d'un rôle de facilitateur (facilitator). Au-delà de cette orthodoxie toutefois, la politique proposée présente quelques dispositions saillantes.

En référence à la Constitution namibienne, elle rappelle tout d'abord les rôles de l'État. Garant du bien-être des Namibiens (Welfare of the People), celui-ci doit veiller à ce que chaque citoyen ait, à des conditions justes et raisonnables, un droit d'accès aux équipements et services publics conformément à la loi ${ }^{5}$. Il doit également assurer un développement durable qui préserve le «patrimoine » écologique national pour les générations présentes et futures. La WASP entérine ces fonctions étatiques en confirmant certaines des responsabilités antérieures du Department of Water Affairs (propriété, préservation, exploitation et contrôle de l'ensemble du patrimoine hydrique, approvisionnement des régions rurales). Les changements portent sur la création d'une société privée nationale pour assumer les responsabilités de production et de commercialisation de l'eau en gros et, en vertu du principe de subsidiarité, sur une plus grande décentralisation de la distribution de proximité et de la gestion des relations avec les usagers. La WASP affirme le principe de «vérité » des prix mais le tempère en admettant que le recouvrement doit porter sur les coûts de fonctionnement puis, progressivement, sur les dépenses d'équipement ; qu'il faut envisager des subventions publiques pour les systèmes ruraux afin de solder la dette sociale de l'État envers les anciens homelands ; qu'il convient enfin de laisser aux municipalités la latitude d'introduire des éléments de péréquation dans leurs propres prix au détail.

\section{LA COMMERCIALISATION DU DEPARTMENT OF WATER AFFAIRS : POUR QUELLE EFFICACITÉ?}

Avec de bons résultats antérieurs, le Department of Water Affairs n'a nullement failli à ses missions. Aussi la réforme ne vient-elle pas sanctionner une crise du service; elle vise, par une commercialisation de l'organisme exploitant, à conformer son statut juridico-institutionnel aux logiques commerciales du secteur dans lequel il intervient et propose, en préservant la propriété de l'État, de confier la desserte en gros à une entité commerciale, la Namibia Water Corporation Ltd ${ }^{6}$.

Ce nouvel agencement doit permettre, selon les consultants, de lever les contraintes budgétaires, tarifaires, gestionnaires et législatives qui limitent aujourd'hui les capacités d'expansion du secteur tout en protégeant le consommateur. En fait, de la clarification institutionnelle et comptable ainsi obtenue, ils attendent surtout deux changements majeurs : la réforme des tarifs de vente de l'eau en gros et la réorganisation du dispositif de régulation.

\section{Vers une politique tarifaire de "vérité » des prix}

La révision des tarifs de l'eau en gros vise à assurer le recouvrement des coûts. Avec un tarif moyen de $\mathrm{N} \$ 1 / \mathrm{m}^{3}$, le revenu collecté par le Department of Water Affairs auprès de ses clients ne couvre pas les dépenses d'équipement et demeure inférieur de $14 \%$ aux coûts d'exploitation sur les sept dernières années. Cette situation est considérée comme mettant gravement en cause la survie du secteur de l'eau : financièrement, parce que le budget national ne peut plus supporter la charge des investissements et écologiquement parce que les subventions masquent le coût réel de l'eau et entretiennent les utilisateurs dans l'illusion d'une eau abondante et bon marché. Orthodoxie économique et préoccupations environnementales conduisent à l'idée que le coût de revient total de l'eau doit être supporté par les utilisateurs finaux, ceux-ci étant alors à même d'apprécier cette denrée rare à son « juste prix » et d'adapter leurs comportements au profit d'une allocation plus efficace de la ressource. Il s'agit donc de diminuer puis de supprimer les subventions publiques, au moins pour les services urbains. En conséquence, des hausses importantes du tarif de l'eau en gros sont inévitables à brève échéance.

À Windhoek, celui-ci pourrait s'élever de $\mathrm{N} \$ 0,95 / \mathrm{m}^{3}$ 
Table2. Évolution des tarifs municipaux de l'eau pour tous les usagers disposant d'un compteur inférieur ou égal à $25 \mathrm{~mm}$ (de 1992 à 1997)*

\section{Illustration non autorisée à la diffusion}

en août 1993 à $\mathrm{N} \$ 2 / \mathrm{m}^{3}$ avant la fin de la décennie et sera intégralement répercuté sur les tarifs de la municipalité. Pour économiser l'eau, celle-ci procède, dans le même temps, à une refonte mesurée de ses barèmes : ayant introduit une tarification progressive en 1991, elle en a révisé chaque année la structure en abaissant progressif les plafonds des tranches de consommation. En outre, deux mesures complémentaires ont été adoptées : l'instauration de tarifs « pénalisants » pour les plus gros usagers domestiques et celle de quotas pour les autres consommateurs (administrations et entreprises). En août 1996, la municipalité envisageait ainsi des quotas calculés sur la consommation moyenne de 1995 diminuée de $30 \%$ et des pénalités de $100 \%$ du tarif en cas de dépassement (Windhoek Aloe, ${ }^{\circ} 5.2,1996$ ).

La mise en œuvre de telles mesures n'a toutefois pas la simplicité que leur confère le message fortement martelé de la pénurie menaçante. Ainsi, c'est moins la disponibilité absolue en eau qui pose problème dans l'immédiat que sa répartition. Or, l'introduction des tranches tarifaires pénalisant les plus gros consommateurs résidentiels en 1991 a montré que si, globalement, la consommation domestique avait été réduite, celle de certaines familles aisées demeurait insensible à cette incitation. Par ailleurs, toute diminution de la consommation a des répercussions négatives sur le budget municipal, dont les revenus issus de la vente d'eau représentent environ $14 \%$ de ses ressources ordinaires depuis le début de la décennie 90 (Kim 1995). Enfin, les amendements tarifaires ne doivent pas revêtir un caractère trop répulsif sous peine de rebuter les gros consommateurs et les investisseurs, qui pourraient privilégier d'autres localisations urbaines en Afrique australe.

\section{Une conception " importée » de la régulation des services publics}

Le second effet majeur de la réforme est d'externaliser et de centraliser la fonction de régulateur pour l'ensemble des services publics commercialisés, entreprises de droit privé propriétés de l'État qui remplissent des missions de service public ${ }^{7}$. Il est ainsi prévu d'instaurer une régulation indépendante par la création d'un organisme centralisé (Public Utilities Commission), libre de toute interférence politique arbitraire, dont les compétences, fixées par la loi, s'étendraient à la protection des consommateurs, à la surveillance des tarifs, au contrôle de l'efficacité des entreprises de service monopolistiques, aux arbitrages entre ces dernières et les autres agents. Ce mécanisme de surveillance s'avère nécessaire puisque la réforme ne supprime pas le quasi monopole national de production de l'eau en gros ; conformément à la tradition anglo-saxonne, la compagnie et l'État en sont écartés au profit d'une commission indépendante.

Ce dispositif classique fait toutefois abstraction des caractéristiques géographiques et démographiques de la Namibie : dans un pays si peu peuplé ( 1,4 million d'habitants), à l'élite si restreinte numériquement (en raison, notamment, du désastreux héritage de l'apartheid dans le domaine de l'éducation des Noirs ${ }^{8}$ ) et si peu contestée politiquement (du fait de l'écrasante domination de la SWAPO, légitimée par la lutte armée contre la puissance 


\section{Jaglin - Le service d'eau potable à Windhoek}

coloniale sud-africaine), l'indépendance de la régulation et, plus généralement, celle des cadres de la future société paraît bien illusoire. Sera-t-elle mieux assurée par une réorganisation institutionnelle qui, sur le fond, ne change ni la dérive politique du recrutement des hauts cadres, laquelle répond - d'abord ? - à la nécessité de placer les héros de la guerre d'indépendance, ni le fait que les cadres noirs susceptibles d'occuper, aujourd'hui, les postes de commandement, ont été formés en exil dans des universités étrangères et sont tous issus du même sérail politique. Les connivences ainsi tissées, l'anémie des contre-pouvoirs, les transferts de légitimité entre fonctions antérieure (rôle dans la guerre) et actuelle (poste de responsabilité civil) ne peuvent que contrecarrer l'objectif d'une régulation indépendante.

Dans ce contexte, il faut s'interroger : faute d'autonomie, une régulation externe et centralisée est-elle néanmoins plus susceptible de remplir correctement ses fonctions, c'est-à-dire de suppléer une concurrence inexistante, qu'une régulation intégrée dans l'entreprise, placée sous l'autorité directe du pouvoir politique et investie d'une mission de service public (au sens français) ? Ce débat est, on le sait, à forte composante idéologique, ce qui n'empêche nullement la circonspection. Sachant que la gestion d'un système de régulation, quel qu'en soit le modèle, est toujours d'une grande complexité en raison de la somme considérable d'informations qu'elle requiert, l'examen de la situation namibienne suscite la réflexion suivante : les compétences localisées dans les postes de commandement technique des administrations publiques, dont l' " africanisation " a surtout concerné les fonctions politiques et les emplois peu qualifiés, sont très largement détenues par un personnel blanc bien formé. Une autorité régulatrice interne aux entreprises commercialisées, lesquelles conservent en partie l'ancien personnel, ne disposerait-elle donc pas plus aisément des informations (notamment celles qui ont été accumulées dans les exercices antérieurs) et des compétences pour les traiter qu'une nouvelle instance, dont le « peuplement » sera nécessairement soumis à des préoccupations politiques ? Dans le premier cas, on peut imaginer que l'appartenance à une même entreprise forge une solidarité qui transcende les éventuelles réticences de communication entre les nouvelles élites dirigeantes noires et les professionnels blancs. Dans le second cas en revanche, on ne peut pas négliger, en raison des caractéristiques du recrutement de la commission, la survenue de perturbations dans le fonctionnement des canaux de trans- mission des données et dans les mécanismes d'acquisition de ces dernières qui amplifient le traditionnel phénomène de dissymétrie de l'information, car si alliances et collusion il y a, elles s'établiront entre les cadres des sociétés commercialisées et ceux de la commission de régulation (ce qui n'exclut donc pas les possibilités de capture du régulateur) et non entre ces derniers et les niveaux intermédiaires du commandement technique, véritables producteurs et détenteurs de l'information. La nomination d'une instance « indépendante » ne suffit pas, en soi, à régler la difficile question d'une régulation satisfaisante et l'adaptation au contexte namibien de cette disposition controversée pose des problèmes spécifiques qui laissent rêveurs quant à l'assurance affichée par l'étude technique.

\section{LA RÉFORME ET LES DYNAMIQUES URBAINES}

L'intérêt de la situation namibienne ne tient donc ni dans son orientation idéologique ni dans l'« emballage » institutionnel proposé pour le futur dispositif. Il est de pouvoir confronter les attendus de cette réforme stéréotypée aux urgences politiques d'un pays bien spécifique. La transition post-apartheid exige en effet des réformes visant à reformuler les liens entre pouvoirs publics et édifice social recomposé, au-delà des clivages raciaux, à forger de nouveaux compromis entre compétitivité et solidarité au sein d'une société profondément divisée : or, les mutations proposées sont étonnamment discrètes sur ces nécessaires redéfinitions, s'en remettant au contraire à une conception anachronique des inégalités sociales et à une appréhension dépassée des dynamiques territoriales.

\section{PERCEPTION DES INÉGALITÉS ET RÉPONSES INSTITUTIONNELLES À L'INJUSTICE SOCIALE}

\section{Un principe : \\ la fin des péréquations inter-régionales}

Dans le souci d'atteindre la "vérité » des prix, l'évolution amorcée au début des années 90 pour uniformiser les tarifs de l'eau sur le territoire est à présent critiquée en raison des subventions croisées qui l'alimentent. En effet, les coûts réels d'exploitation diffèrent sensiblement d'un réseau régional à l'autre et, dans un souci d'équité sociale 
et territoriale, l'augmentation annuelle des tarifs a été assortie, après l'indépendance, de l'application d'un plafond visant à réduire progressivement les écarts de prix. Ainsi, tandis que certains réseaux exploités par le Department of Water Affairs n'assurent pas le recouvrement de leurs coûts de fonctionnement, les tarifs de l'eau en gros à Windhoek sont supérieurs de $15 \%$ à $20 \%$ à ces mêmes coûts, les habitants de la capitale contribuant ainsi à une redistribution inter-régionale.

La réforme prend nettement parti contre celle-ci. Cette remise en cause n'est pas propre à la Namibie, les économistes faisant valoir que les transferts tarifaires nuisent à l'allocation optimale des ressources collectives en dissimulant à certaines catégories de clients le surcoût réel du service qui leur est offert, l'utilité collective maximale étant au contraire obtenue par une tarification au coût marginal (Stoffaës 1995).

Dans un pays dont tout le système économico-politique a consisté, pendant plusieurs décennies, à surexploiter les périphéries nationales au profit d'un centre aujourd'hui très bien équipé, il y a néanmoins urgence à trouver les moyens de façonner une nouvelle cohésion sociale et spatiale, notamment par l'extension aux secteurs défavorisés du territoire et de la société (dont les répartitions géographiques ne coïncident plus forcément) des réseaux de services élémentaires. La péréquation tarifaire n'est certes pas le seul moyen de contribuer à cette solidarité, mais de nombreuses expériences ont montré qu'elle pouvait atteindre cet objectif sans soulever d'oppositions radicales. À l'inverse, la ponction qu'exercent sur les contribuables les transferts fiscaux qui ont à présent la préférence des économistes a une forte visibilité : rarement bien tolérée, elle suscite de fortes résistances, y compris dans des contextes de " reconstruction nationale » favorables à des manifestations de solidarité. La récente révolte des contribuables blancs de certaines banlieues riches sud-africaines témoigne ainsi avec éclat de la faible acceptabilité sociale d'une solidarité fiscale explicite (Pienaar 1996).

\section{La survalorisation de la coupure ville-campagne}

Reste qu'il faut bien tenir compte des disparités considérables de développement et de niveaux de vie. En raison de la géographie des inégalités issues de l'apartheid, la réforme propose de scinder le pays en distinguant la «zone commerciale » (regroupant les villes et bourgs) du monde rural, auxquels seront appliqués deux régimes institutionnels et gestionnaires différents. Les zones rurales, envers lesquelles l'État a une «dette sociale", demeureront gérées par le Department of Water Affairs, tandis que dans les villes seront introduits les principes d'une gestion plus commerciale, axée sur la notion d'efficacité productive sinon de profit, puisque celui-ci devrait être strictement encadré9.

Afin de leur restaurer toute leur compétitivité, les activités rentables sont ainsi déconnectées des activités déficitaires qui peuvent alors bénéficier de différentes aides (à la production ou à la consommation), issues de transferts fiscaux plutôt que tarifaires. Cette solution, conforme aux dogmes économiques contemporains, conduit à dissocier les préoccupations sociales et politiques de la logique entrepreneuriale du service de l'eau : «Si la compagnie doit être commercialisée en vertu de réels principes commerciaux, alors elle ne doit pas être responsable des conséquences sociales de sa politique tarifaire et des augmentations de prix projetées » (MAWRD, 1994, p. 2.7).

En proposant cette séparation, la réforme répartit, apparemment sans ambiguïté, les responsabilités entre la nouvelle compagnie, investie d'une logique commerciale dans les aires économiquement développées, et un Department of Water Affairs, amaigri et restructuré, administrant la solidarité nationale envers le monde rural. Toutefois, en négligeant la fraction croissante des ruraux qui s'agglomèrent sur les franges urbaines de la Namibie " développée ", ce partage des tâches ne règle pas le sort d'un troisième segment de la demande, celui des citadins très pauvres pour lesquels la nécessité d'aides à l'intégration est tout aussi forte.

\section{LES « PÉRIPHÉRIES » URBAINES : UNE CLIENTĖLE OUBLIÉE}

L'intégration métropolitaine de populations et de territoires urbains auparavant ségrégués est un enjeu essentiel de la période post-apartheid: les réseaux techniques comme les services auxquels ils servent de supports peuvent y contribuer. Toutefois, la dichotomie établie, pour la gestion de l'eau, entre un monde «commercial » et un monde rural nuit à la crédibilité de cet objectif en relé- 
guant dans un entre-deux indistinct les enjeux qui se rapportent aux «périphéries » urbaines : quartiers précaires (légaux et illégaux) des anciennes municipalités et villes des ex-homelands, artificiellement distinguées de leur arrière-pays. Une fraction non négligeable et croissante des usagers de l'eau est ainsi fondue dans un ensemble vague et mal identifié.

À Windhoek, ces périphéries correspondent à des quartiers précaires 10 localisés au nord et à l'est de l'ancienne township de Katutura. Ils ont pour caractéristiques communes d'être de peuplement récent (postindépendance), de regrouper des ménages pauvres, d'offrir des niveaux de desserte qui demeurent, y compris dans les lotissements les mieux consolidés, très inférieurs aux normes de la ville et d'accueillir en nombre croissant des squatters, installés illégalement sur des terrains privés ou publics. Notion multidimensionnelle, l'intégration urbaine de ces quartiers recouvre de nombreux objectifs mais leur desserte en services est à l'évidence l'un d'entre eux. Or, dans le secteur de l'eau, les documents consultés appréhendent mal les conditions d'approvisionnement des clientèles spécifiques ainsi concernées.

L'économie de cette réflexion est surprenante de la part des études sectorielles récentes. Mentionné allusivement dans la WASP, qui souligne l'existence de pauvres urbains et la nécessité de dépenses d'investissement continues dans les villes pour maintenir les taux de couverture actuels (MAWRD/DWA, 1993-b, p. 14), le problème est évoqué en un paragraphe péremptoire dans le WATCOM PROJECT qui réduit les communautés de squatters urbains à « une forme transitoire d'établissements » (MAWRD, 1994, pp. 1-3). Or, à Windhoek au moins, le problème n'est pas seulement celui des nécessaires investissements, il est aussi celui de la formulation d'une offre en services accessible aux citadins pauvres. Il ne s'agit pas non plus d'imaginer un dispositif transitoire, mais bien de concevoir une offre durablement différenciée qui réponde, en termes institutionnels et techniques, à la concentration géographique de citadins aux aspirations et aux pouvoirs d'achat trop disparates pour relever de services identiques. La question de la pauvreté urbaine n'est ni un problème subsidiaire, ni un écueil provisoire. Faute d'en convenir, les documents préparatoires ne fournissent aucun élément de réflexion sur les moyens dont disposeront les futurs organismes gestionnaires pour concevoir et faire fonctionner à la fois des dispositifs unitaires assurant un lien de citoyenneté entre tous les citadins et une offre en service différenciée, adaptée notamment aux pauvres pour lesquels des prestations standards sont inaccessibles.

Cette approche lacunaire a des raisons proprement namibiennes. Il est ainsi patent que les politiques sectorielles enregistrent et répercutent un décalage croissant entre les héritages de l'apartheid à l'indépendance, l'analyse qu'en ont forgée les milieux politiques nationaux et les réalités actuelles : les disparités criantes entre villes et campagnes demeurent d'actualité, mais elles ne sont qu'une des manifestations des très profondes inégalités qui frappent la société namibienne et dont la répartition géographique est en cours d'évolution. C'est ainsi que des disparités tout aussi graves affectent les villes et, compte tenu des flux migratoires, tendent à s'aggraver. La césure majeure oppose toujours les Blancs riches aux Noirs pauvres mais, d'une part elle se double de nouveaux clivages", d'autre part son tracé recoupe de moins en moins la traditionnelle frontière ville-campagne qui tend à masquer, dans les représentations, des lignes de fracture dont les conséquences paysagères sont pourtant de plus en plus visibles dans la capitale. Sans doute la discrétion du monde politique namibien sur les efforts à consentir en faveur des usagers citadins très pauvres traduit-elle aussi d'autres enjeux : la dette d'un État qui s'est enrichi au détriment de ses périphéries, l'urgence d'une politique de "réparation ", mais aussi et surtout ? - le poids des Ovambo au sein de l'appareil politique et la redistribution qui en résulte au profit d'un groupe ethnique majoritairement rural.

À ces explications, il faut ajouter d'autres arguments qui ne sont nullement spécifiques à ce pays. En effet, si les études consacrées, depuis une dizaine d'années, à la pauvreté dans les villes en développement sont nombreuses, elles ne sont pas accompagnées de la généralisation d'un appareillage statistique adapté à la restitution des inégalités intra-urbaines (Stephens 1996). Au contraire, les catégories et les échelles d'analyse retenues (l'ensemble urbain ou, dans les villes d'apartheid, les grandes unités raciales : Blancs, Métis et/ou Indiens, Noirs) agrègent des données qui sont surtout utilisées pour comparer les villes entre elles ou opposer ces dernières aux zones rurales. Ce faisant, elles exagèrent la coupure ville-campagne, que surpasse au contraire une large diffusion de la pauvreté, et elles masquent les effets d'une polarisation croissante des sociétés citadines. 


\section{FLUX $n^{\circ} 30$ Octobre-Décembre 1997}

Ainsi, rappeler que le revenu moyen par habitant de Windhoek est très supérieur à la moyenne nationale (N\$ 11000 contre N\$ 3000 ) est utile à condition de souligner aussi que les inégalités dans la capitale sont très grandes et grossièrement mesurées : N\$27000/capita dans la ville blanche contre N\$ 4 300/capita dans la township noire de Katutura (CSO/NPC 1996, p. 229)12. Certes, la capitale namibienne dispose d'un niveau d'équipement très supérieur à celui de nombreuses villes africaines avec $99 \%$ des ménages disposant d'eau à moins de $5 \mathrm{mn}$ de chez eux (CSO/NPC 1996, p. 226) ; encore faut-il préciser que ces statistiques ignorent les 30000 habitants qui se partagent le réseau de robinets collectifs dans les périphéries où de nombreux citadins vivent en-dessous du seuil de pauvreté (plus de $50 \%$ des ménages à Katutura au début de la décennie 90), dans des conditions de dénuement souvent dramatiques (Keulder 1994). Enclave de richesse, Windhoek rassemble un nombre croissant de ménages très pauvres et présente des écarts de revenu accrus sans que les outils statistiques disponibles permettent d'expliquer précisément les causes, les manifestations et la répartition spatiale de la pauvreté en son sein. En conséquence, les politiques urbaines travaillent avec des représentations de la société citadine artificiellement lissées, qui masquent une différenciation de la demande en services et logements qu'elles ne prennent pas suffisamment en compte.

Mal mesurée et cartographiée, cette différenciation n'est pas non plus bien traitée par les organismes gestionnaires publics qui, comme partout, ont au contraire formulé des politiques d'offre fondées sur la standardisation des prestations, tant par souci technique de simplifier les schémas de desserte que par préoccupation idéologique d'égalité de traitement des usagers. En conséquence, et jusqu'à une date récente, ces organismes ont été peu demandeurs de données susceptibles de fonder des distinctions entre des sous-catégories de clientèle et de justifier des traitements spécifiques. En dépit de réalités urbaines souvent plus contrastées qu'ailleurs, la reconnaissance d'une différenciation durable de la demande urbaine ne semble pas non plus, en Namibie, à l'ordre du jour.

\section{SOCIÉTÉ PRIVÉE, ÉTAT ET MUNICIPALITÉS, QUELS PARTENARIATS?}

Les partis pris que nous avons soulignés ont deux conséquences importantes pour la gestion du service. Tout d'abord, ils ne permettent pas de poser clairement la question des mécanismes de redistribution et du rôle des municipalités dans les solidarités territoriales. Ensuite, ils entravent la mise au point de solutions adaptées aux différentes catégories de clientèles qui, pour présenter des exigences de qualité et des pouvoirs d'achat très hétérogènes, n'en sont pas moins prêtes à payer pour accéder à de l'eau potable.

\section{Des mécanismes de redistribution à inventer}

Si les auteurs de la réforme n'envisagent pas d'adaptation technico-institutionnelle de l'offre pour tenir compte de la clientèle urbaine la plus pauvre, ils admettent en revanche que, même dans les aires économiquement développées du pays, le principe de recouvrement des coûts risque d'écarter du service une fraction des usagers à laquelle ils recommandent d'accorder des aides sociales fournies par les services ad hoc dans chaque aire urbaine. Ayant rejeté cette question en direction des pouvoirs publics, les documents ne s'interrogent ni sur l'échelle à laquelle la redistribution doit être effectuée, ni sur ses modalités, ni même sur les incidences qui, selon les cas, pourraient en résulter sur les consommations et l'évolution des clientèles.

Qui, des municipalités ou de l'État, devra prendre en charge cette redistribution dès lors que les aires de compétence du Department of Water Affairs sont circonscrites aux espaces ruraux et que les principes commerciaux de la future société privée excluent les tarifs subventionnés de l'eau en gros ? Compte tenu de l'importance politique des charges ainsi transférées et de l'ampleur économique qu'elles pourraient, à moyen terme, représenter, il est frappant de constater que pas une ligne des documents préparatoires de la réforme n'explicite la redistribution des responsabilités ainsi occasionnée ni n'évoque, d'ailleurs, les relations à établir, dans les territoires urbains entre l'État, la nouvelle compagnie et ses clients municipaux.

Assistera-t-on à un partage des tâches, l'État assumant la redistribution en direction du monde rural et les municipalités la solidarité dans les villes ? De quels revenus ces dernières disposent-elles pour cela, sachant que les prélèvements directs sur les activités économiques reviennent en très grande majorité à l'État ? Compte tenu des dynamismes démographiques et de la fixation d'une part croissante de la pauvreté à la périphérie des villes, les instances gestionnaires urbaines ne seront-elles pas rapi- 
Jaglin - Le service d'eau potable à Windhoek

dement débordées par l'ampleur des transferts nécessaires? Quels peuvent être leurs arbitrages entre une péréquation tarifaire limitée au service d'eau, des subventions croisées entre plusieurs services (pratique que la municipalité a jusqu'à présent refusée ${ }^{13}$ ) et une redistribution assise sur des transferts fiscaux?

Qu'en sera-t-il, dans ces circonstances, des articulations à établir, au sein du territoire communal, entre la société de production et de distribution d'eau en gros, le gestionnaire du réseau de proximité et l'État ? Les choix stratégiques des uns et des autres ne seront pas, en effet, sans incidences sur leurs activités respectives. Ainsi, compte tenu de l'étroitesse de sa clientèle urbaine solvable, la Namibia Water Corporation Ltd devra, pour accroître son marché et assurer l'extension de ses activités, viser la demande des nouvelles populations citadines tant dans les extensions périphériques de la capitale que dans les villes « champignons » des provinces septentrionales du pays. Pour cela, elle devra compter, dans un premier temps au moins, avec les mécanismes de subvention à la consommation consentis par les pouvoirs publics, municipalité et/ou État. Inversement, dans un pays qui reste très largement à équiper, où des milliers de gens ne disposent pas encore d'un accès à l'eau potable, les pouvoirs publics ne pourront pas assumer les charges d'aménagement du territoire si la Namibia Water Corporation Ltd confisque à son profit les activités les plus rentables du secteur de l'eau sans redistribuer, en concertation avec les collectivités locales, une partie de ses revenus au profit d'une extension géographique de la desserte.

\section{Gérer la différenciation durable de la demande urbaine}

En ne reconnaissant pas la différenciation de la demande urbaine en eau et, partant, la nécessité d'une offre diversifiée, la réflexion en cours sur le service d'eau potable s'épargne une épineuse question : comment assurer la coexistence durable, sur un même territoire urbain, de prestations répondant à des normes et des modes de gestion différents, éventuellement fournies grâce à des dispositifs techniques distincts, comme les mini-réseaux d'adduction d'eau de certaines périphéries urbaines d'Afrique de l'Ouest (BURGÉAP/Groupe Huit 1994) ? La réticence ancienne des organismes gestionnaires à l'égard de cette diversification de l'offre, éventuellement assortie d'une autonomisation technique de certains segments de production/distribution de l'eau, peut reposer sur la crainte d'une atomisation gestionnaire nuisible à la cohérence du service ou procéder d'un refus d'assumer les coûts de gestion accrus qui en résulteraient pour l'organisme chargé d'assurer la coordination de l'ensemble.

Comme dans de nombreux pays, où la séparation des institutions gestionnaires chargées de la gestion de l'eau en ville et dans les campagnes s'accommode, en revanche, d'une reconnaissance de la spécificité des clientèles rurales, la Namibie s'apprête à entériner cette distinction au détriment d'une juste appréciation de la différenciation de la demande intra-urbaine. Ainsi, divers documents évoquent avec insistance la nécessité d'innover dans la façon de concevoir et de gérer la distribution d'eau en milieu villageois : ils prévoient des séminaires de formation à la communication et à l'animation communautaire pour des agents spécialement chargés d'assumer des responsabilités dans les villages ; ils insistent sur les qualités de dialogue et d'écoute qui sont nécessaires pour conformer l'offre à une demande évolutive. Si l'hydraulique villageoise motive l'introduction de nouveaux savoirs et de nouveaux codes de conduite dans la formation des techniciens, rien de similaire n'est en revanche prévu pour les villes. Le dialogue entre les techniciens municipaux et les squatters urbains serait-il d'emblée plus aisé qu'avec les villageois? Le changement d'attitude des premiers envers la demande des seconds et la révolution professionnelle qu'il implique sont-ils moins urgents ? Confrontés, sur le terrain, à des collectifs résidentiels dont les modalités d'insertion en ville remettent en cause leur approche traditionnelle du service, les ingénieurs locaux s'efforcent pourtant d'adapter leur pratique professionnelle. Non sans pragmatisme, ils ont amorcé une concertation avec les habitants et réfléchi aux moyens d'assouplir les normes en vigueur pour favoriser des dispositifs de desserte évolutifs. Ce faisant, ils se heurtent aux rigidités des réglementations héritées, au conservatisme des représentations, notamment celles du pouvoir politique, et les documents de réforme, peu diserts sur les besoins de ces clientèles urbaines spécifiques, ne semblent guère en mesure de les soutenir dans leur démarche.

\section{CONCLUSION}

Les mutations du secteur de l'eau en Namibie ont de quoi laisser perplexe. Si l'indépendance politique et le déman- 
tèlement de l'apartheid ne peuvent qu'accélérer les réformes dans les grands services urbains, celles-ci semblaient pouvoir être mûries et programmées sur le moyen terme, afin de préparer leur insertion dans une société ébranlée par les bouleversements des dernières années. En effet, pragmatique et performant au regard d'autres dispositifs de production et distribution d'eau africains, le système namibien n'appelait pas une urgente remise en cause de son architecture institutionnelle. En revanche, il se trouve confronté à deux impérieuses nécessités : gérer la pénurie d'eau d'une façon plus durable et économe ; assumer la desserte d'une nouvelle clientèle « difficile » tant dans les périphéries des principales villes que dans les nouveaux centres urbains.

Or, la réforme prend le parti d'une refonte du dispositif institutionnel qui n'apporte pas de solution aux deux impératifs précédents. Si elle réaffirme le principe d'un puissant contrôle public sur la production de l'eau en gros, elle concentre l'effort sur la modernisation des structures juridiques et leur adaptation aux exigences d'efficacité de gestion par l'instauration d'une société privée à capitaux publics. L'introduction des principes du marché dans le fonctionnement de ce service peut certes favoriser sa souplesse et sa capacité d'adaptation, mais les documents préparatoires prennent fort peu en considération des logiques politiques et sociales pourtant susceptibles de gripper le déroulement de l'opération et d'en dénaturer les objectifs. Ils font ainsi l'économie d'une réflexion sur le partage de l'eau, la gestion de la pénurie et la place de ce service public dans la société namibienne : il est bien possible que la future société privée, recentrée sur les segments de clientèle les plus rentables et rivée à ses principes de vérité des coûts, soit plus efficace économiquement, mais rien n'est réglé quant aux modes et moyens d'assurer un accès correct à l'eau pour ceux qu'a exclus l'apartheid et que marginalise actuellement le chômage.

Apparemment, les arrangements locaux existant dans les anciennes municipalités ne sont pas modifiés et deviennent même la règle. Toutefois, le statu quo est illusoire car faute d'aborder le problème de la pauvreté, de l'extension géographique et sociale du service, des relations entre les municipalités et la nouvelle société privée, la réforme impute, de facto, de nouvelles responsabilités aux pouvoirs locaux, très inégalement armés pour les assumer. Si la réforme est appliquée telle quelle dans les mois à venir, elle aura précédé la clarification des rôles que d'aucuns estiment nécessaire (Lorrain 1995) : la façon dont l'entreprise privée assumera des responsabilités de service public n'est pas plus explicitée que le financement des charges sociales résultant du contexte économique comme de l'introduction plus spécifique des contraintes commerciales dans le service de l'eau. Le gouvernement namibien semble quant à lui fort peu empressé dans cette phase finale : si un opérateur dénommé Namibia Water Corporation Ltd fonctionne en 1997, l'examen parlementaire de ses statuts et fonctions, initialement prévu en mars 1996, est depuis reporté. La situation est ainsi d'une grande confusion puisque la société qui négocie et passe des contrats n'a aucune réalité juridique et ne peut, de ce fait, obtenir des emprunts internationaux. La confidentialité entourant ces tergiversations étatiques ne permet pas d'établir avec certitude les raisons du blocage mais ce dernier résulte, très vraisemblablement, de profondes divergences au sein du pouvoir central.

Sigles

BURGÉAP : Bureau d'Étude de Géologie Appliquée et d'Hydrologie CSO : Central Statistical Office DWA : Department of Water Affairs

MAWRD : Ministry of Agriculture, Water and Rural Development NPC : National Planning Commission

SADC : Southern Africa Development Community

SWAPO : South West African People's Organisation 


\section{Jaglin - Le service d'eau potable à Windhoek}

NOTES

1. Sauf indication contraire, les données factuelles sur le service d'eau sont extraites des documents suivants : City of Windhoek, City Engineer's Department, Van der Merwe B., 1994 : MAWRD/DWA. $1993-$ a.

2. $\mathrm{NS}=1,2 \mathrm{FF}$ en 1996

3. Hypothèses municipales (City of Windhoek, City Engineer's Department, Van der Merwe B., 1994, p. 5).

4. Cette entité renvoie au périmètre d'étude du Central Area Water Master Plan.

5. The Constitution of the Republic of Namibia, [1990], Windhoek, The Ministry of Information and Broadcasting, p. 52.

6. Pour un descriptif détaillé de la réforme et de la future compagnie, voir : MAWRD, 1994-a et -b.

7. Il en existe déjà deux en Namibie : Namibia Post and Telecom Holdings Limited, département du ministère des Postes et Télécommunications commercialisé en 1992, et la SWAWEK (SWA Water and Electricity Company Pty Limited), créée en 1960.

8. La Banque mondiale rapporte ainsi qu'en 1989 , la moyenne des effectifs par classe primaire était de 37 dans l'Ovamboland avec seulement $2 \%$ d'enseignants qualifiés. Par comparaison, ces chiffres étaient de 13 et $92 \%$ respectivement dans les zones blanches (World Bank, 1992, p. 7).

9. L'étude préliminaire recommande que la Compagnie ne puisse réaliser de profits au delà de son seuil de rentabilité, afin de maintenir des tarifs bas à moyen et long termes et de rendre la réforme socialement acceptable. Elle n'indique pas, toutefois, les moyens d'atteindre cet objectif.

10. Etablissements noirs pauvres. L'adjectif précaire qualifie ici le type de bâti dominant et l'équipement sommaire des quartiers. Il ne s'applique pas au statut foncier des occupants, qui peut être légal - durable ou temporaire - ou illégal.

11. Déclassement de certains " petits Blancs » et promotion rapide d'une élite noire peu nombreuse mais puissante : voir par exemple les villas de standing construites ces dernières années dans Katutura ancien.

12. Ces ratios ne concernent que les ménages recensés ; ils ne prennent pas en compte les extensions illégales les plus récentes de la capitale où sont concentrés les ménages de squatters.

13. Les excédents de la vente d'électricité sont néanmoins reversés sur le compte des services non marchands (non remunerative services), qui sont surtout financés par la taxe sur la valeur des propriétés. La surtaxe sur les tarifs de l'électricité sert ainsi à modérer la pression fiscale sur les propriétaires mais n alimente pas de subventions croisées à proprement parler. Une exception notable à cette règle a toutefois été consentie en 1995 , le conseil municipal autorisant par permission spéciale qu'une partie des "surplus " (unappropriated surplus) aille aux services d'assainissement déficitaires.

\section{RÉFÉRENCES}

BURGÉAP/Groupe Huit. 1994. Etude méthodologique de l'alimentation en eau potable des zones périurbaines africaines. Rapport final, version définitive, Paris, BURGÉAP, $95 \mathrm{p}$.

City of Windhoek, City Engineer's Department, Van der Merwe Ben. 1994. Water demand management in Windhoek. Namibia, Septembre, revised, 22 pp. + annexures.

CSO/NPC. 1996. Living Conditions in Namibia. Basic Description with Highlights. Main Report, Windhoek, CSO-NPC, 285 p.

Keulder Ch. 1994. Urban Women and Self-Help Housing in Namibia: a Case-Study of Saamstaan Housing Cooperative. Windhoek, NEPRU, 21 p., (NWP ${ }^{\circ} 42$ ).

Kim K.-H. 1995. Preliminary Mission Report. 10 February, $6 \mathrm{p}$.

Lorrain D. 1995. “Introduction : l'extension du marché ", in Lorrain D. et Stoker G. (dirs.), La privatisation des services urbains en Europe. Paris, La Découverte, pp. 9-30.

MAWRD. 1994-a. WATCOM PROJECT. Draft. Phase I. Windhoek, Ernst \& Young, April.

MAWRD. 1994-b. WATCOM PROJECT: Phase 2. Windhoek, Ernst \& Young, July to November, 7 volumes.
MAWRD/DWA. 1993-a. Central Area Water Master Plan: Phase I. Windhoek, CES/LCE/WCE. 11 volumes.

MAWRD/DWA. 1993-b. A Digest of the Water Supply and Sanitation Sector Policy of the Government of Namibia. Windhoek, DWA, November, 36 p. + annexes.

Melber H. 1996. Urbanisation and Internal Migration: Regional dimensions in Post-Colonial Namibia. Windhoek, NEPRU, 27 p. (NWP n ${ }^{\circ} 48$ ).

NEPRU. 1997. The Namibian Economy, ${ }^{\circ}$ 10, June.

Pienaar J. 1996. «Les difficultés dans l'attribution de logements au niveau local », communication aux journées PIRVilles sur l'Afrique du Sud, 12-13 novembre, 20 p., dactyl.

Stephens C. 1996. "Healthy cities or unhealthy islands? The health and social implications of urban inequality ", in Environment and Urbanization, vol. 8, $\mathrm{n}^{\circ} 2$, October, pp. 930.

Stoffaës Ch. 1995. Services publics : question d'avenir. Paris, Odile Jacob/La Documentation française.

Windhoek Aloe. Monthly Newsletter. Tous les numéros de 1991 à juillet 1997.

World Bank. 1992. Namibia. Poverty Alleviation with Sustainable Growth. Washington, The World Bank. 